\title{
Aplicação da Decomposição em Valores Singulares e Análise de Componentes Independentes em dados de fMRI
}

\author{
Liliane R. da Silva Luiz R. de A. Lopes* \\ Programa de Pós-Graduação em Sistemas e Computação- DIMAp-CCET-UFRN \\ Campus Universitário, Lagoa Nova, 59078-970, Natal, RN \\ E-mail: liliane@ppgsc.ufrn.br, ranyer.lopes@gmail.com \\ Valcir J. da C. Farias \\ Marcus P. C. da Rocha \\ Programa de Pós-graduação em Matemática e Estatística-ICEN-UFPA \\ Rua Augusto Corrêa, 01, Belém, Pará \\ E-mail: valcir@ufpa.br; mrocha@ufpa.br
}

\begin{abstract}
RESUMO
A técnica de imagens funcionais por ressonância magnética (fMRI) é usada para localizar e analisar diferentes aspectos da atividade cerebral humana. Durante uma experiência de fMRI, uma sequência de imagens é adquirida enquanto o paciente desempenha tarefas específicas. No entanto, as alterações no contraste das imagens não são grandes, o que dificulta uma inspeção visual direta, tornando necessária a utilização de algoritmos computacionais de identificação dessas áreas. Nesse sentido, investigou-se o método de Análise de Componentes Independentes (ICA), com o diferencial da aplicação da Decomposição em Valores Singulares (SVD) nos dados já pré-processados utilizando dados artificiais, para avaliar a sensibilidade e a especificidade do método. As curvas ROC (Receiver Operating Characteristic) indicam que o método tiveram um melhor desempenho após a utilização da SVD. Além disso, ICA foi aplicada a um conjunto de dados reais de fMRI onde um indivíduo normal realizou um experimento prédefinido.
\end{abstract}

Palavras-chave: $f M R I$, decomposição de valores singulares, análise de componentes independentes, ROC, FastICA.

\section{Introdução}

Em um simples procedimento fMRI o sujeito é submetido a estímulos variados enquanto sucessivas imagens vão sendo adquiridas. O scanner MR detecta as atividades funcionais cerebrais a partir de mudanças nos níveis oxigenação do sangue, as quais causam pertubações no campo magnético aplicado. Estas alterações na taxa de oxigenação no sangue são conhecidas como contraste ou efeito BOLD (Blood Oxigenation Level Dependent), e podem ser modeladas através da função resposta hemodinâmica (Hemodynamic Response Function).

As imagens obtidas em um experimento fMRI, geralmente, possuem uma baixa relação sinal ruído (SNR). Desse modo, torna-se necessário algum tipo de pré-processamento para a filtragem do ruído presente no sinal. As técnicas de pré-processamentos servem para diminuir a maioria dos ruídos, como batimentos cardíacos, pois o ruído é um componente indesejável e com variabilidade irrelevante, presente no sinal associado à resposta BOLD, [1] .

A ausência de pré-processamento pode invalidar o poder de detecção de áreas cerebrais consideradas ativas ou inativas em um experimento.

Nesse sentido, aplicou-se o método de ICA, com o diferencial da aplicação da SVD nos dados já pré-processados utilizando dados artificiais, para avaliar a sensibilidade e a especificidade do método. As curvas ROC indicam que o método teve um melhor desempenho após a utilização da SVD, [3]. Além disso, ICA foi aplicada a um conjunto de dados reais de fMRI onde um indivíduo normal realizou um experimento pré-definido.

\section{Análise de Componentes Independentes}

O principal objetivo da ICA é encontrar uma representação linear de dados não gaussianos, minimizando a dependência estatística entre eles de forma que os componentes resultantes sejam estatisticamente independentes, ou tão independentes quanto possível. O que

* Sem vínculo com nenhuma Instituição 
diferencia a ICA das outras técnicas existentes é, justamente, o fato dela trabalhar com componentes que são ao mesmo tempo não gaussianos e estatisticamente independentes.

Existem diversos algoritmos que são utilizados para a implementação do princípio estatístico, nesse momento, já determinado. $\mathrm{O}$ algoritmo rápido de ponto fixo (FastICA) é um dos mais utilizados e que apresenta uma melhor estimativa das componentes independentes, [2].

\subsection{Decomposição em Valores Singulares}

Antes de aplicarmos os algoritmos para estimar as componentes independentes, vamos aplicar a SVD como uma etapa de redução da dimensão dos dados, objetivando uma redução significativa no custo computacional.

A grande dimensão dos dados de fMRI, normalmente no domínio do espaço, e além disso os algoritmos de ICA são, na maioria das vezes, de computação intensiva, [4]. Por esse motivo, antes de aplicar ICA vamos realizar uma redução de dimensão dos dados usando o SVD. Para isso, as matrizes ortogonais desempenham um papel importante neste artigo.

Seja $X_{m \times n}$, com $m \geq n$. Então existem matrizes ortogonais $U_{m \times m}$ e $V_{n \times n}$ tais que $\boldsymbol{X}=\boldsymbol{U} \boldsymbol{D} \boldsymbol{V}^{T}$, onde as matrizes $U$ e $V$ são matrizes ortogonais e $D$ é uma matriz diagonal que contém os autovalores de $X$. As colunas das matrizes $U$ e $V$, são chamados autovetores à esquerda e à direita de $X$, respectivamente. Aqui $U$ e $V$ podem ser vistos como os vetores de base que abrangem os padrões especiais e sequências temporais, respectivamente.

A ideia da etapa de redução dos dados antes do ICA é reduzir a dimensão das matrizes a serem utilizadas como insumos para algoritmos de ICA.

\subsection{Algoritmo Rápido de Ponto Fixo (FastICA)}

Os algoritmos baseados em iterações de ponto fixo denominados FastICA [2] são diferenciados pela abordagem e pela função custo utilizada, eles visam encontrar componentes independentes através da maximização da negentropia. Foram desenvolvidas duas versões do FastICA: a primeira permite a recuperação de todas as fontes (multi-unit) e a segunda encontra um dos componentes (one-unit). Ambas podem utilizar qualquer função custo não-paramétrica para estimar a negentropia das fontes.

O algoritmo FastICA procura encontrar uma direção, ou seja, um vetor cuja projeção maximiza a função $\mathrm{J}_{\mathrm{G}}(\mathrm{W})$, ou seja, uma aproximação da negentropia dada por:

$$
\mathrm{J}_{\mathrm{G}}(\mathrm{W})=\left[\mathrm{E}\left[\mathrm{G}\left(\mathrm{W}^{\mathrm{T}} \mathrm{X}(k)\right]-\mathrm{E}[\mathrm{G}(b)]\right]^{2}\right.
$$

$\mathrm{G}($.$) é qualquer função não quadrática e b$ é uma variável gaussiana de média zero e variância unitária. Na prática, as esperanças E[.] são substituídas por médias amostrais. A regra de treinamento do algoritmo consiste de um método que estima os componentes um a um.

\section{Conclusão}

Os resultados obtidos nos dados sintéticos demonstraram um desempenho satisfatório quando utilizada a função $\mathrm{G}_{4}$, porém, ao aplicar o método nos dados reais os resultados se mostraram pouco expressivos. No entanto, a utilização do SVD mostrou-se muito eficiente na otimização do processamento computacional, sem o SVD o tempo computacional é incomparavelmente maior.

\section{Referências}

[1] D. B. de Araujo, Sobre neuroimagens funcionais por magnetoencefalogra e ressonância magnética: novos métodos e aplicações, Tese (Doutorado). USP, FFCLRP., 2002.

[2] D. Bamber, The Area above the Ordinal Dominance Graph and the Area below the Receiver Operating Characteristic Graph. J. Math Psychol 12, 387-415, 1975.

[3] J. R. Beck, and E. K. Shultz, The Use of Relative Operating Characteristic (ROC) Curves in Test Performance Evaluation. Arch Pathol Lab. Med. 110, 13-20,1986.

[4] A. Hyvarinem, One Unit Contrast Function for Independent Component Analysis: A Statistical Analysi. Proc. IEEE Workshop on Neural Network for Signal Processing, p.388397, Florida.

\footnotetext{
* Sem vínculo com nenhuma Instituição
} 